\title{
Glucocorticoid-Related Molecular Signaling Pathways Regulating Hippocampal Neurogenesis
}

\author{
Christoph Anacker*, 1,2,3, Annamaria Cattaneo ${ }^{4}$, Alessia Luoni ${ }^{5}$, Ksenia Musaelyan', Patricia A Zunszain $^{1,2}$, \\ Elena Milanesi ${ }^{4}$, Joanna Rybka ${ }^{6}$, Alessandra Berry ${ }^{7}$, Francesca Cirulli ${ }^{7}$, Sandrine Thuret ${ }^{3}$, Jack Price $^{3}$, \\ Marco A Riva ${ }^{5}$, Massimo Gennarelli ${ }^{4,8}$ and Carmine M Pariante \\ 'Section of Perinatal Psychiatry and Stress, Psychiatry and Immunology (SPI-Lab), Institute of Psychiatry, Department of Psychological Medicine, \\ King's College London, London, UK; ${ }^{2}$ National Institute for Health Research, Biomedical Research Centre for Mental Health, Institute of Psychiatry \\ and South London and Maudsley NHS Foundation Trust, London, UK; ${ }^{3}$ Centre for the Cellular Basis of Behaviour (CCBB), Institute of Psychiatry, \\ King's College London, London, UK; ${ }^{4}$ Department of Biomedical Sciences and Biotechnology, University of Brescia, Brescia, Italy; ${ }^{5}$ Department of \\ Pharmacological and Biomolecular Sciences, University of Milan, Milan, Italy; ${ }^{6}$ Department of Biochemistry, Nicolaus Copernicus University in \\ Toruń, Bydgoszcz, Poland; ${ }^{7}$ Department of Cell Biology and Neuroscience, Istituto Superiore di Sanita, Rome, Italy; ${ }^{8}$ Genetics Unit, \\ Fatebenefratelli, Giovanni di Dio, Brescia, Italy
}

\begin{abstract}
Stress and glucocorticoid hormones regulate hippocampal neurogenesis, but the molecular mechanisms underlying their effects are unknown. We, therefore, investigated the molecular signaling pathways mediating the effects of cortisol on proliferation, neuronal differentiation, and astrogliogenesis, in an immortalized human hippocampal progenitor cell line. In addition, we examined the molecular signaling pathways activated in the hippocampus of prenatally stressed rats, characterized by persistently elevated glucocorticoid levels in adulthood. In human hippocampal progenitor cells, we found that low concentrations of cortisol ( $100 \mathrm{nM}$ ) increased proliferation $(+16 \%)$, decreased neurogenesis into microtubule-associated protein 2 (MAP2)-positive neurons ( $-24 \%)$ and doublecortin (Dcx)positive neuroblasts $(-21 \%)$, and increased differentiation into SIO0 3 -positive astrocytes $(+23 \%)$. These effects were dependent on the mineralocorticoid receptor (MR) as they were abolished by the MR antagonist, spironolactone, and mimicked by the MR-agonist, aldosterone. In contrast, high concentrations of cortisol $(100 \mu \mathrm{M})$ decreased proliferation ( $-17 \%)$ and neuronal differentiation into MAP2-positive neurons (-22\%) and into Dcx-positive neuroblasts ( $-27 \%)$, without regulating astrogliogenesis. These effects were dependent on the glucocorticoid receptor (GR), blocked by the GR antagonist RU486, and mimicked by the GR-agonist, dexamethasone. Gene expression microarray and pathway analysis showed that the low concentration of cortisol enhances Notch/Hessignaling, the high concentration inhibits TGFB-SMAD2/3-signaling, and both concentrations inhibit Hedgehog signaling. Mechanistically, we show that reduced Hedgehog signaling indeed critically contributes to the cortisol-induced reduction in neuronal differentiation. Accordingly, TGF $\beta$-SMAD2/3 and Hedgehog signaling were also inhibited in the hippocampus of adult prenatally stressed rats with high glucocorticoid levels. In conclusion, our data demonstrate novel molecular signaling pathways that are regulated by glucocorticoids in vitro, in human hippocampal progenitor cells, and by stress in vivo, in the rat hippocampus.

Neuropsychopharmacology (20 I3) 38, 872-883; doi:I 0.1038/npp.20 I2.253; published online 16 January 2013
\end{abstract}

Keywords: depression; prenatal stress; stem cells; Hedgehog signaling; MR; GR

\section{INTRODUCTION}

The molecular signaling mechanisms by which glucocorticoid hormones regulate hippocampal neurogenesis are unknown. Stress increases circulating levels of endogenous glucocorticoids (Pariante and Lightman, 2008; Anacker et al, 2011a), which in turn decrease adult hippocampal neurogenesis (David et al, 2009; Anacker et al, 2011b; Gould

*Correspondence: Dr C Anacker, Section of Perinatal Psychiatry and Stress, Psychiatry and Immunology (SPI-Lab), Institute of Psychiatry, Department of Psychological Medicine, King's College London, 125 Coldharbour Lane, London SE5 9NU, UK, Tel: +44 (0)20 78480352 , Email: Christoph.Anacker@kcl.ac.uk

Received 7 August 2012; revised 28 October 2012; accepted 20 November 2012; accepted article online preview 6 December 2012 et al, 1991; Surget et al, 2011). Importantly, impaired hippocampal neurogenesis in rodents has recently been shown to contribute to the development of depressive symptoms in response to stress (Snyder et al, 2011), suggesting that the glucocorticoid-induced reduction in neurogenesis may indeed contribute to the pathogenesis of major depression. Discovering the molecular signaling pathways by which glucocorticoids regulate hippocampal neurogenesis is, therefore, crucial to indentify potential targets for novel antidepressant treatments that counteract stress-induced neurobiological abnormalities.

Endogenous glucocorticoids (cortisol in humans and corticosterone (CORT) in rodents) act on two intracellular receptors: the mineralocorticoid receptor (MR), which has high affinity for endogenous glucocorticoids and is already 
activated by low, basal cortisol concentrations; and the glucocorticoid receptor (GR), which has low affinity for cortisol and is thus predominantly activated by high cortisol concentrations, as they occur upon stress and in depression (Anacker et al, 2011a; Pariante et al, 2008; Pariante and Miller, 2001). We and others have demonstrated that stress and GR activation consistently decrease hippocampal neurogenesis in vitro and in vivo (Anacker et al, 2011b; Kim et al, 2004; Fujioka et al, 2006; Gould et al, 1991; Mayer et al, 2006; David et al, 2009). In contrast, the role of the MR in neurogenesis is less clear, as some studies have reported a decrease in proliferation upon MR activation (Wong and Herbert, 2006; Montaron et al, 2003), while others have found an increase (Fischer et al, 2002; Gass et al, 2000). Previous studies have investigated candidate genes involved in the stress- and glucocorticoid-induced reduction in neurogenesis (Datson et al, 2010; Sippel et al, 2009; Xi et al, 2010; Matrisciano et al, 2011). However, a complete analysis of the intricate signaling mechanisms that mediate the effects of glucocorticoids on the hippocampus is lacking.

To address this issue, we have first analyzed the effects of low (ie, MR-related) and high (GR-related) cortisol concentrations on an immortalized human hippocampal progenitor cell line, our established in vitro model of human neurogenesis in which we can determine the state of proliferation and neuronal differentiation by the addition and removal of growth factors (Anacker et al, 2011b; Zunszain et al, 2011). Using pharmacological agonists and antagonists for the MR and the GR, in combination with immunocytochemistry and whole genome expression analysis, we have dissected MR- and GR-mediated regulation of neurogenesis and astrogliogenesis. We have then conducted whole genome expression analysis of the rat hippocampus in animals subjected to prenatal stress, a well established animal model of depression in which glucocorticoid levels are elevated and hippocampal neurogenesis is decreased (Lemaire et al, 2000; Fumagalli et al, 2007). Our study reveals novel molecular signaling pathways regulated consistently by cortisol in human hippocampal progenitor cells and by stress in the rat hippocampus.

\section{MATERIALS AND METHODS}

\section{Cell Culture}

The immortalized, multipotent human fetal hippocampal progenitor cell line HPC03A/07 (provided by ReNeuron Ltd, UK) was used for all experiments (Anacker et al, 2011b). HPC03A/07 cells proliferate in the presence of growth factors (EGF, FGF) and 4-hydroxytamoxifen (4-OHT), while differentiation is initiated upon growth factor and 4-OHT removal. Detailed information on this cell line and culture conditions can be found in the Supplementary Materials.

\section{Drugs}

All drugs and reagents were purchased from Sigma-Aldrich (St Louis, MO) unless otherwise stated. Growth factors EGF and bFGF were purchased from Peprotech (London, UK). Purmorphamine was from Millipore, UK. Aldosterone, dexamethasone, hydrocortisone, RU486 and spironolactone were dissolved in $100 \%$ ethanol. Purmorphamine was dissolved in 100\% DMSO. Bromodeoxyuridine (BrdU) was dissolved in phosphate-buffered saline fresh before use.

\section{Proliferation Assay}

Progenitor cell proliferation was assessed as previously described (Anacker et al, 2011b) Briefly, HPC03A/07 cells were plated on 96-well plates (Nunclon, Roskilde, Denmark) at a density of $1.2 \times 10^{4}$ cells per well and cultured for $72 \mathrm{~h}$ in the presence of growth factors and 4-OHT to maintain proliferation. The synthetic nucleotide $5^{\prime}$-BrdU, $10 \mu \mathrm{M}$ ) was added to the media $4 \mathrm{~h}$ before treatment cessation. Cells were fixed with $4 \%$ paraformaldehyde for $20 \mathrm{~min}$ at roomtemperature.

\section{Differentiation Assay}

Differentiation was assessed as previously described (Anacker et al, 2011b). Briefly, cells were cultured in the presence of growth factors and 4-OHT for 3 days, and subsequently washed and cultured in media without growth factors and 4-OHT for further 7 days to allow differentiation. For these experiments, cells were treated during the initial proliferation phase and the subsequent differentiation phase, or only during the proliferation phase, or only during the differentiation phase. At the end of the total incubation time (10 days) cells were fixed as described above.

\section{Immunocytochemistry}

Proliferation was assessed by immunolabelling for BrdU (rat anti-BrdU, Serotec, Oxford, UK). Differentiation into young and mature neurons was assessed by immunocytochemistry for Dcx (rabbit anti-Dcx, 1:1000, Abcam, Cambridge, UK) and microtubule-associated protein-2 (MAP2) (mouse anti-MAP-2[HM], 1:500, Abcam), respectively. Astrogliogenesis was evaluated by immunocytochemistry for S100 calcium-binding protein $\beta$ (S100 $\beta$ ) (rabbit antiS100 $\beta, 1: 500$, DAKO, Glostrup, Denmark). Synaptic markers were detected by staining for synaptophysin (mouse anti-synaptophysin, 1:100, Assaydesigns) and Homer1 (rabbit anti-Homer1, 1:100, Synaptic Systems). Cells were also stained for the hippocampal granule-cell-marker, Prospero homeobox protein 1 (Prox1) (mouse anti-Prox1, $1: 100$, Abcam). Hoechst 33342 dye was used to label all cells. The number of Dcx-, MAP2-, S100 $\beta$ - and BrdUpositive cells over total Hoechst 33342-positive cells was determined to assess neurogenesis, astrogliogenesis, and progenitor proliferation (see Supplementary Materials).

\section{GR Transactivation Assay}

To measure GR transactivation in HPC03A/07 cells upon cortisol treatment, nuclear protein extracts were obtained using a commercially available nuclear extraction kit (Active Motif, Rixensart, Belgium). GR binding to a consensus glucocorticoid response element DNA sequence was analyzed using the enzyme-linked immunosorbent assay-based TransAM GR method (Active Motif), according to the manufacturer's instructions. 


\section{Ethics Statement}

All animal handling and experimental procedures were performed in accordance with the EC (EEC Council Directive 86/609 1987), the Italian legislation on animal experimentation (Decreto Legislativo 116/92), and the National Institutes of Health Guide for the Care and Use of Laboratory Animals. All efforts were made to minimize animal suffering and to reduce the number of animals used.

\section{Prenatal Stress Study}

Nulliparous adult female (body weight 230-260 g) and male (400 g) Sprague-Dawley rats were pair-housed with a samesex conspecific with food and water available ad libitum $\left(21 \pm 1{ }^{\circ} \mathrm{C}, 60 \pm 10 \%\right.$ relative humidity, reversed $12 / 12 \mathrm{~h}$ light/dark cycle). After 10 days, rats were mated for $24 \mathrm{~h}$ and individually housed immediately thereafter. Pregnant females were randomly assigned to delivery control (ctrl) and prenatal stress (PNS) conditions. PNS consisted of restraining pregnant dams in a transparent Plexiglas cylinder $(7.5 \mathrm{~cm}$ diameter, $19 \mathrm{~cm}$ length) under bright light $(6.5001 \times)$ for $45 \mathrm{~min}$ three times daily during the last week of gestation. PNS sessions were separated by $2-3 \mathrm{~h}$ intervals and conducted at varying periods of the day to reduce habituation. Control rats were left undisturbed. Male offspring from control and PNS groups were killed at postnatal day (PND) 62 for whole hippocampal dissection ( $n=7$ for each group) and trunk blood collection $(n=5$ for each group).

\section{CORT Analysis}

Upon killing, trunk blood samples were collected individually in potassium EDTA tubes $(1.6 \mathrm{mg}$ EDTA/ml blood, Sarstedt, Germany). CORT was measured using a commercially available radioimmunoassay kit containing 125Iodine labeled CORT (MP Biomedicals, CA). Assay sensitivity was $0.125 \mathrm{mg} / \mathrm{dl}$. Vials were counted for $2 \mathrm{~min}$ in a gamma-scintillation counter (Packard Minaxi Gamma counter, Series 5000).

\section{RNA Isolation, Microarray Processing, Quality Control}

RNA of human progenitor cells and rat hippocampal tissue was isolated using RNeasy mini kit (Qiagen, Crawley, UK) and subsequently DNase treated (Ambion, Warrington, UK). RNA was reverse transcribed using GeneAtlas $3^{\prime} \mathrm{IVT}$ Express Kit (Affymetrix, Santa Clara, CA).

Gene expression microarray assays were performed on three independent experiments using Human Genome U219 Array Strips (for the gene expression analyses in cells) and Rat Gene 1.1 T Array Strips (for the gene expression analyses in rat) on the Affymetrix GeneAtlas platform (http//www.affymetrix.com/support/technicalmanual/expression_manual_affy). Partek Genomics Suite 6.6 software was used for data visualization, statistical testing of affymetrix CEL files and quality control. Data quality was assessed using histograms of signal intensities, scatter plots, and hierarchical clustering of samples. All samples passed the criteria for hybridization controls, labeling controls and $3^{\prime} /$ $5^{\prime}$ Metrics. Robust MultiChip Average method was used for background correction and Quantiles normalization. Summarization was performed using a median polish algorithm (Tukey 1977) (see also Supplementary Materials).

\section{Pathway Analysis}

Pathway analysis for progenitor cells and hippocampi of PNS rats was conducted with Pathway Studio Software 5.0 (Ariadne, Lausanne, Switzerland), using the standard Gene Set enrichment analysis, originally developed by the Broad Institute (http://www.broad.mit.edu/gsea/) (Mootha et al, 2003). This algorithm uses a correlation-weighted Kolmogorov-Smirnov statistic on all gene expression changes and computes pathway enrichment scores by considering gene set membership information, gene list ranking and gene-gene dependencies that reflect real biology. We used $P<0.005$ as enrichment $P$-value cutoff for pathway analysis in human hippocampal progenitor cells, and $P<0.05$ as enrichment $P$-value cutoff for pathway analysis in PNS rat hippocampi.

\section{Quantitative Real-time PCR}

RNA from human cells and rat hippocampal tissue was reverse transcribed with Superscript III (Invitrogen) before quantitative Real-Time PCR (qPCR) using the SYBR Green method (Supplementary Materials).

\section{Statistical Analysis}

All statistical analyses for immunocytochemistry and qPCR were performed with GraphPad Prism 4.03 (GraphPad, La Jolla) on independent biological replicates (indicated as ' $n$ '). One-way ANOVA with Newman-Keuls post hoc test was used for multiple comparisons among the treatment groups. Student's $t$-test was used to compare means of two independent treatment groups. $P$-values $<0.05$ were considered significant. Data are presented as mean \pm s.e.m.

ANOVA was used in the gene expression microarray to assess the effects of cortisol treatment on progenitor cells and the effects of PNS in rats. To investigate the effects of cortisol on progenitor cells, a three linear contrast was performed using Partek Genomic Suite 6.6 (cortisol $100 \mathrm{~nm}$ vs vehicle; cortisol $100 \mu \mathrm{M}$ vs vehicle; cortisol $100 \mu \mathrm{M} v s$ cortisol $100 \mathrm{~nm}$ ) with a maximum filter of $P<0.05$ and a minimum absolute fold-change cutoff of 1.2. Genes that passed these criteria were used to build up the Venn diagram (Supplementary Figure 4a, Supplementary Table 3). For both, cortisol-treated cells and adult PNS rats, the cluster diagrams were built up using genes fulfilling the criteria of $P<0.01$ and 1.2 minimum absolute foldchange (Supplementary Tables 4 and 5; Supplementary Figures 5 and 6).

\section{RESULTS}

\section{Differential MR- and GR-dependent Effects of Cortisol on Progenitor Cell Proliferation}

We first analyzed the effects of a wide range of cortisol concentrations $(1 \mathrm{nM}-100 \mu \mathrm{M})$ on proliferation of human hippocampal progenitor cells after 3 days of treatment, 
using BrdU incorporation and immunocytochemistry (Figure 1a).

Cortisol treatment showed dose-dependent, bimodal effects on proliferation (One-way ANOVA, $P<0.0001$, $\left.F_{1,7}=6.76, n=4\right)$. Specifically, low concentrations (1 nM$100 \mathrm{nM}$ ) increased proliferation (by $16 \%$ at $100 \mathrm{~nm}$, $P=0.002, n=4$; Figure $1 b$, third column), whereas high concentrations $(1 \mu \mathrm{M}-100 \mu \mathrm{M})$ decreased proliferation (by $17 \%$ at $100 \mu \mathrm{M}, P=0.002, n=4$; Figure $1 \mathrm{~b}$, last column). In order to investigate whether MR and GR are involved in these effects, we co-treated cells with cortisol (again $1 \mathrm{nM}-$ $100 \mu \mathrm{M})$ and with the MR antagonist, spironolactone $(1 \mu \mathrm{M})$, or the GR antagonist, RU486 (50 nM). Spironolactone, but not RU486, blocked the increase in proliferation upon treatment with low cortisol concentrations ( $1 \mathrm{nM}-100 \mathrm{nM})$, indicating that this effect is mediated by the MR (Figure 1c). Conversely, RU486, but not spironolactone, blocked the decrease in proliferation upon treatment with high cortisol concentrations $(1 \mu \mathrm{M}-100 \mu \mathrm{M})$ (Figure $1 \mathrm{~d})$, indicating that this effect is mediated by the GR.
To confirm these findings, we also examined whether MR- and GR-agonists would model the differential effects of low- and high-cortisol concentrations on proliferation. Indeed, the MR-agonist, aldosterone ( $1 \mathrm{nM}-1 \mu \mathrm{M})$, increased proliferation (One-way ANOVA, $P=0.02, F_{1,5}=3.27, n=3$; Figure 1e), modeling the effects of low cortisol concentrations. Moreover, the GR-agonist, dexamethasone (10 nM$5 \mu \mathrm{M}$ ), decreased proliferation (One-way ANOVA, $P<0.0001, F_{1,5}=7.66, n=3$; Figure 1f), modeling the effects of high cortisol concentrations and confirming our previous data on decreased proliferation upon dexamethasone treatment $(1 \mu \mathrm{M})$ in the same model (Anacker et al, 2011b).

To further confirm that the higher cortisol concentrations used in our study do not fully saturate the GR, we examined GR transactivation upon cortisol treatment over a wider range of concentrations $(100 \mathrm{~nm}-2 \mathrm{~mm})$. In line with our data on progenitor cell proliferation (described above), low doses of cortisol (100 nM) did not significantly induce GR binding to the DNA, while GR transactivation was dosedependently increased following higher cortisol concentrations a
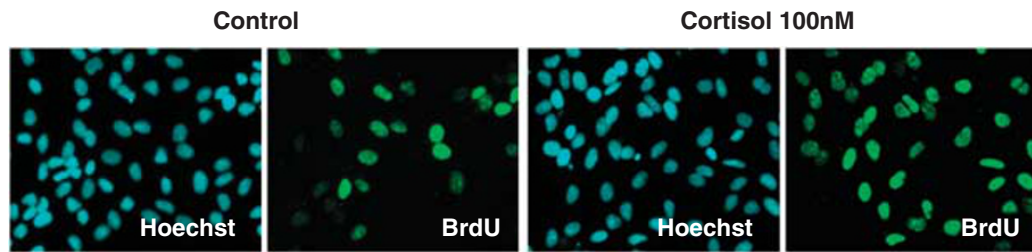

C

b

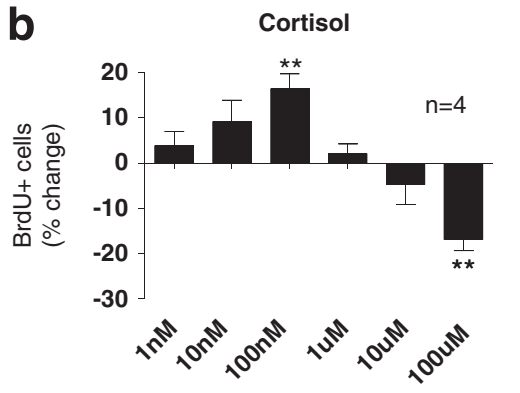

Cortisol + Spironolactone
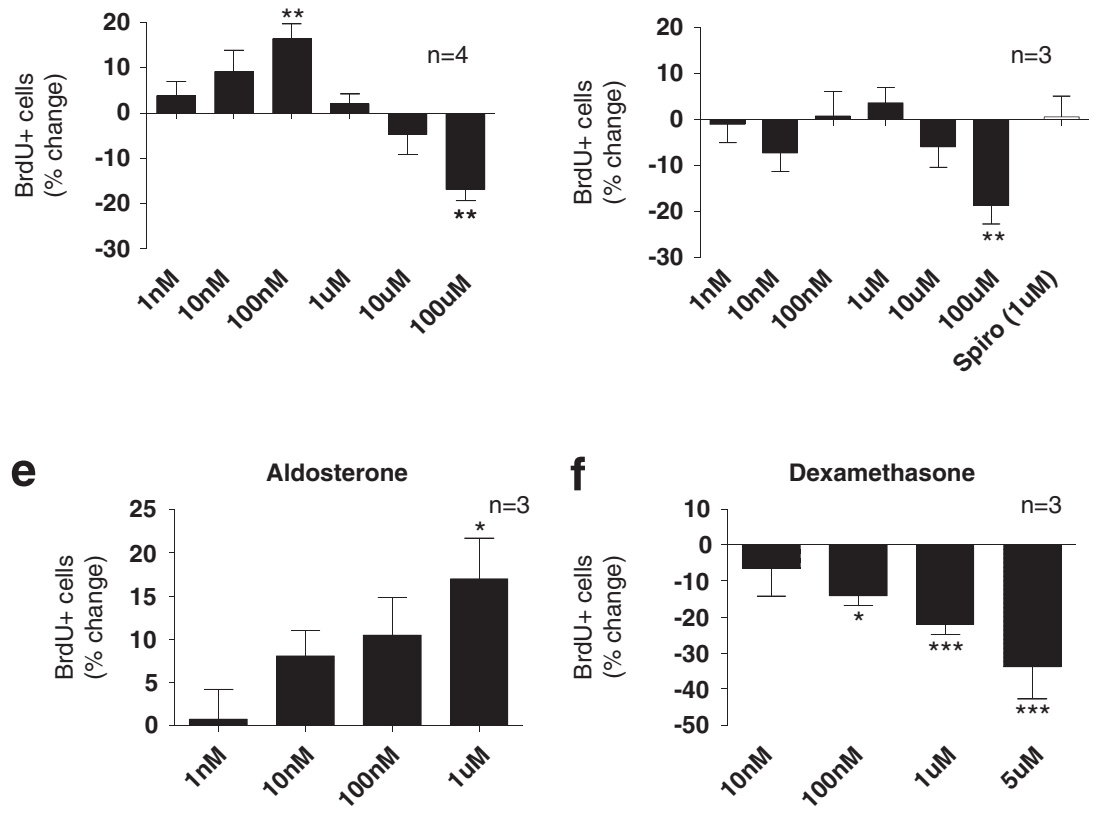

f



Cortisol $100 \mu \mathrm{M}$

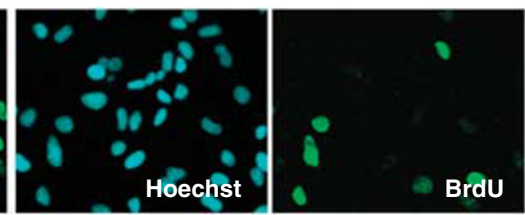

d
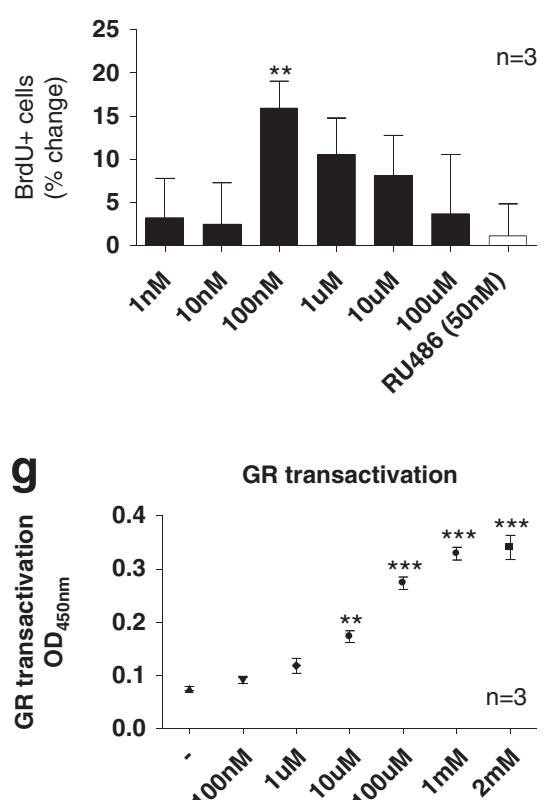

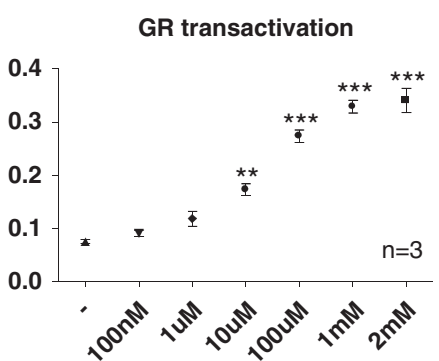

Figure I Differential effects of mineralocorticoid receptor (MR)- and glucocorticoid receptor (GR)-activation on human hippocampal progenitor cell proliferation. 5'-bromodeoxyuridine (BrdU, IO $\mu \mathrm{M}$ ) incorporation and immunocytochemistry was used to assess proliferation of HPC03A/07 cells (a). Cortisol exerts bimodal, dose-dependent effects on proliferation (b). Spironolactone (I $\mu \mathrm{M})$ blocks the increase in cell proliferation upon treatment with low concentrations of cortisol (c). RU486 (50 nM) blocks the decrease in cell proliferation upon treatment with high concentrations of cortisol (I $00 \mu \mathrm{M})$ (d). The MR-agonist, aldosterone (I nM-I $\mu \mathrm{M}$ ), increases cell proliferation (e), while the GR-agonist, dexamethasone ( $10 \mathrm{nM}-5 \mu \mathrm{M})$, decreases cell proliferation (f). GR transactivation is dose-dependently increased by cortisol concentrations (I0 $\mu \mathrm{M}-\mathrm{I} \mathrm{mm})(\mathrm{g})$. Three to four independent experiments were conducted on independent cultures (indicated as $n$ ). In proliferation experiments, four wells were analyzed per treatment condition in each experiment and three random, non-overlapping pictures were analyzed for each well. All data are mean \pm s.e.m. $* P<0.05, * * P<0.0$ I; $* * * P<0.00$ I compared with the vehicle-treated control condition. 
$(10 \mu \mathrm{M}-2 \mathrm{mM})$. Moreover, the concentration of $100 \mu \mathrm{M}$ cortisol did not saturate the GR, as transactivation could be further increased by concentrations of up to $2 \mathrm{~mm}$ (Figure 1g). Concentrations of cortisol higher than $2 \mathrm{~mm}$ induced cell death.

\section{MR- and GR-dependent Effects of Cortisol on Differentiation into Neurons}

To examine the effects of cortisol on neuronal differentiation, cells were treated with two cortisol concentrations selected for predominant MR activation (100 nM) or GR activation $(100 \mu \mathrm{M})$, as described above. Cells were cultured for 3 days under proliferation conditions and subsequently allowed to differentiate by removal of growth factors and 4OHT for subsequent 7 days. Immunocytochemistry for MAP2 and Dcx was used to visualize mature neurons and young neuroblasts, respectively (Figure 2a, top panels; Supplementary Figure 1a).

When cells were treated only during the proliferation phase ( 3 days) but not during the subsequent differentiation phase (7 days), both cortisol concentrations decreased neuronal differentiation. Specifically, the low concentration $(100 \mathrm{~nm})$ decreased MAP2-positive neurons by $-24 \%$ $(P=0.005, n=3$; Figure $2 \mathrm{~b})$. As expected, this effect was counteracted by the MR antagonist, spironolactone $(1 \mu \mathrm{M})$, and mimicked by the MR-agonist, aldosterone $(1 \mu \mathrm{M})$ $(-21 \%, P=0.01, n=3$; Figure $2 b)$. Similar effects were observed when we counted the number of Dcx-positive neuroblasts (Supplementary Results, Supplementary Figure 1b). The high cortisol concentration $(100 \mu \mathrm{M})$ also decreased MAP2-positive neurons by $-22 \%(P=0.002$, $n=3$; Figure $2 \mathrm{c}$ ). This effect was abolished by RU486 $(50 \mathrm{nM})$, and mimicked by dexamethasone $(1 \mu \mathrm{M})(-27 \%$, a
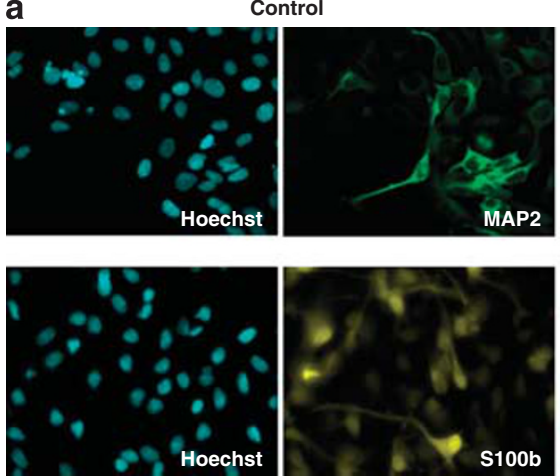

Cortisol 100nM
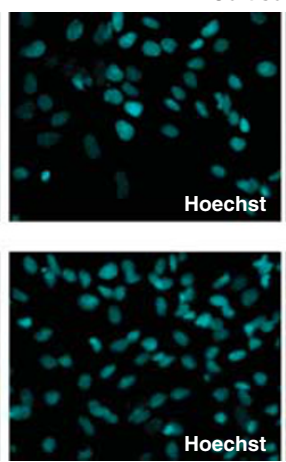
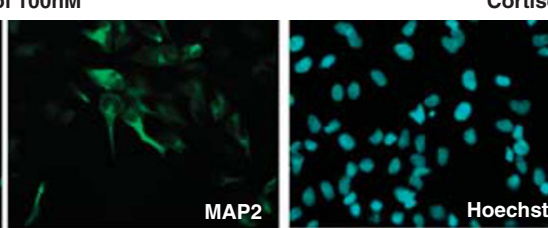

Cortisol $100 \mu \mathrm{M}$
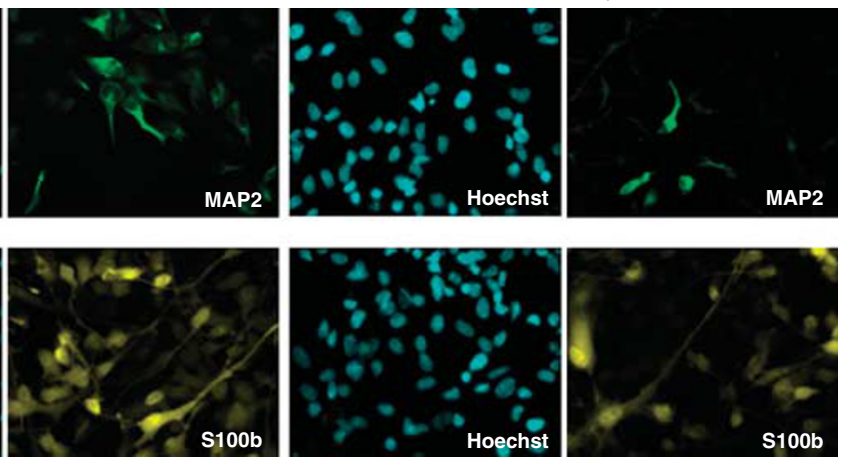

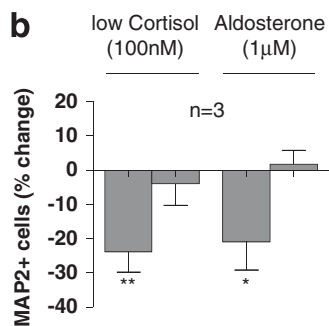

Spironolactone $(1 \mu \mathrm{M})$

f low Cortisol Aldosterone

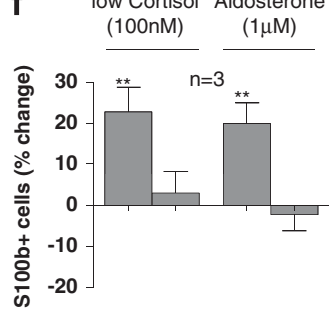

Spironolactone $(1 \mu \mathrm{M})$

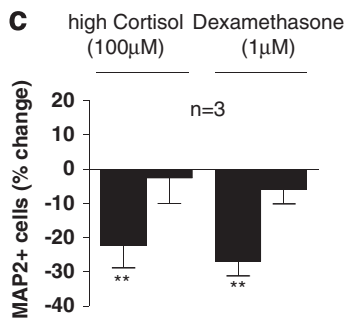

$\mathrm{RU} 486$ (50nM) - $+\quad+$



$\mathrm{RU} 486(50 \mathrm{nM})$


Figure $\mathbf{2}$ Low and high concentrations of cortisol exert differential MR- and GR-dependent effects on neurogenesis and astrogliogenesis. Immunocytochemistry for microtubule-associated protein 2 (MAP2) and SI 00 calcium-binding protein $\beta$ (SI 00ß) (a). If treated only during the proliferation phase, the low cortisol concentration ( $100 \mathrm{nM})$ and aldosterone (I $\mu \mathrm{M})$ decrease the number of MAP2-positive neurons. Both effects are counteracted by spironolactone (I $\mu \mathrm{M})(\mathrm{b})$. The high cortisol concentration ( I00 $\mu \mathrm{M})$ and dexamethasone (I $\mu \mathrm{M})$ also decrease the number of MAP2-positive neurons. These effects are both counteracted by RU486 (50 nM) (c). No effects on the number of MAP2-positive neurons are observed when cells are treated only during the differentiation phase ( $d$, e). the low cortisol concentration (I00 nM) and aldosterone (I $\mu M$ ) increase the number of SI00 $\beta$-positive astrocytes. Both effects are counteracted by spironolactone (I $\mu \mathrm{M})$ (f). The high cortisol concentration (I00 $\mu \mathrm{M})$ and dexamethasone (I $\mu \mathrm{M})$ do not significantly alter the number of SIOOK-positive astrocytes (g). No effects are observed on the number of SIO0 $\beta$-positive astrocytes when cells are treated only during the

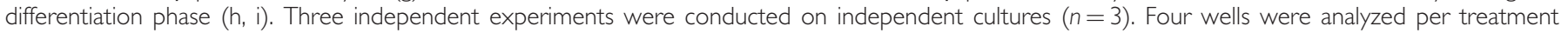
condition in each experiment and three random, non-overlapping pictures were analyzed for each well. All data are mean \pm s.e.m. $* P<0.05$, $* * P<0.01$, compared with the vehicle-treated control condition. 
$P=0.001, n=3$; Figure 2c). Again, similar effects were observed when we counted the number of Dcx-positive neuroblasts (Supplementary Results, Supplementary Figure 1c). These data demonstrate that MR activation and GR activation both decrease neuronal differentiation.

When cells were treated continuously during both, the proliferation and the differentiation phase, we observed similar effects on MAP2- and Dcx-positive cells (Supplementary Results, Supplementary Figure 2a-d). In contrast, treatment only during the differentiation phase, but not during the preceding proliferation phase, did not exert any significant effects (Figure $2 \mathrm{~d}$ and e, Supplementary Figure $1 \mathrm{~d}$ and e), supporting the notion that MR- and GRactivation during the proliferation phase is necessary and sufficient to reduce neuronal differentiation.

All experiments above were conducted by allowing cells to differentiate for 7 days. In order to elucidate whether these newborn neurons continue to further develop into hippocampal granule neurons that express synaptic markers, we analyzed the expression of synaptic markers and hippocampal granule-cell markers after longer periods of differentiation ( 5 weeks), when most cells have already fully differentiated into MAP2-positive neurons. Indeed, differentiated HPC03A/07 neurons express the synaptic markers, synaptophysin and homer1, as well as the hippocampal granule-cell-marker, Prox1 (Karalay et al, 2011) (Supplementary Figure 3).

\section{MR- and GR-dependent Effects of Cortisol on Differentiation into Astrocytes}

Next, we wanted to test whether the MR- and GR-mediated decrease in neuronal differentiation, described above, is associated with a cortisol-induced shift of progenitor cell fate from neuronal- to astrocyte-differentiation. We thus treated cells with cortisol and analyzed the number of S100 $\beta$-positive astrocytes (Figure 2a, bottom panels). Treatment with the low cortisol concentration (100 nM) and aldosterone $(1 \mu \mathrm{M})$ only during the proliferation phase increased the number of S100 $\beta$-positive astrocytes (by $23 \%$, $P=0.003, n=3$; and by $20 \%, P=0.004$, respectively; Figure 2f). Both effects were abolished by spironolactone $(1 \mu \mathrm{M})$ (Figure $2 \mathrm{f})$. In contrast, the high cortisol concentration $(100 \mu \mathrm{M})$ and dexamethasone $(1 \mu \mathrm{M})$ did not change the number of $\mathrm{S} 100 \beta$-positive astrocytes (Figure $2 \mathrm{~g}$ ), demonstrating for the first time that MR activation, but not GR activation, induces astrocyte differentiation of human hippocampal progenitor cells. However, when interpreting our data it needs to be considered that the high cortisol concentration also activates the MR (deKloet and Derijk 2004). Notably, this high concentration induced a small increase in $S 100 \beta$-positive astrocytes in the presence of RU486 (Figure 2g, second column). Therefore, the lack of effect suggests that predominant GR activation may inhibit the MR-mediated increase in astrogliogenesis (as also suggested by our pathway analysis, see below), resulting in the net effect of no changes.

When cells were treated continuously during proliferation and differentiation, similar effects on S100 $\beta$-positive astrocytes were observed (Supplementary Results, Supplementary Figure $2 \mathrm{e}$ and $\mathrm{f}$ ). Finally, and similar to the effects on neuronal differentiation, treatment only during the differentiation phase, but not during the preceding proliferation phase, did not exert any significant effects (Figure $2 \mathrm{~h}$ and $\mathrm{i}$ ), supporting the notion that MR activation during the proliferation phase is necessary and sufficient to induce astrogliogenesis.

Taken together, our results indicate that MR activation increases hippocampal progenitor cell proliferation while concomitantly shifting cell fate from neuronal- toward astrocyte differentiation. In contrast, GR activation decreases proliferation and neuronal differentiation and counteracts the MR-induced increase in astrogliogenesis.

\section{Regulation of Molecular Signaling Pathways by Low and High Cortisol Concentrations}

Having discovered such profound effects of MR- and GRmediated cortisol action on hippocampal neurogenesis, we wanted to establish the molecular signaling pathways implicated in these effects. We focused specifically on the signaling mechanisms during the proliferation phase, as treatment during this phase was necessary and sufficient to reduce neurogenesis and to increase astrogliogenesis, as described above. We analyzed gene expression changes after 12 hours of cortisol treatment, because our initial real-time PCR analysis had shown the most significant effects of cortisol on two selected glucocorticoid-regulated genes (FKBP5, FOXO1) specifically at this time point (Supplementary Figure 4). In this Results section we present the results of the pathway analysis, as this analysis best captures the complex signaling mechanisms induced by low- and high-concentrations of cortisol (presented in Table 1). The complete gene expression analysis is presented in Supplementary Figure 5a and 6, Supplementary Table 3 and 4 and in the Supplementary Results.

The low cortisol concentration $(100 \mathrm{~nm})$ regulated 36 pathways $(P<0.005)$. Of particular relevance is the activation of Notch- and Hairy/Enhancer of Split (Hes)-signaling, which is crucially involved in changing neuronal- to astroglial cell fate (Kageyama and Ohtsuka, 1999). Activation of this pathway may thus potentially be involved in the above described MR-mediated shift from neurogenesis to astrogliogenesis upon treatment with low cortisol concentrations in our experiments.

The high cortisol concentration $(100 \mu \mathrm{M})$ regulated five pathways $(P<0.005)$, among which TGF $\beta$-SMAD2/3-signaling was downregulated and FOXO3A signaling was activated (Table 1). Both pathways have been implicated in neurogenesis, depression, and glucocorticoid action (Shull et al, 1995; Graciarena et al, 2010; Seuntjens et al, 2009; Musil et al, 2011), and their regulation is thus consistent with decreased progenitor proliferation in our experiments. However, opposite to the effect of the low concentration, the high cortisol concentration decreased Notch/Hes-signaling (at a lower significance level: $P=0.04$ ). This could possibly be explained by high cortisol concentrations predominantly activating the GR, thereby inhibiting the MR-mediated increase in these signaling pathways present with low concentrations.

Finally, both low and high cortisol concentrations significantly regulated seven pathways. Inhibition of Hedgehog signaling is thereby particularly relevant, because 
Cortisol, MR, GR and neurogenesis

C Anacker et al

Table I Pathways Regulated by Cortisol in Human Hippocampal Progenitor Cells

Pathways regulated by cortisol $100 \mathrm{nM}$



Pathways regulated by cortisol $100 \mu \mathrm{M}$

\begin{tabular}{lcc}
\hline Pathway name & Change & P-value \\
\hline T-cell activation & + & 0.0001 \\
TGF $\beta$ receptor $\rightarrow$ SMAD2/3 signaling & - & 0.001 \\
GF signaling & + & 0.002 \\
GFR $\rightarrow$ FOXO3A signaling & + & 0.002 \\
Endocytosis & + & 0.004
\end{tabular}

Pathways regulated by cortisol $100 \mathrm{nM}$ and $100 \mu \mathrm{M}$

\begin{tabular}{|c|c|c|c|}
\hline Pathway name & Change & P-value Corticosterone $100 \mathrm{nM}$ & P-value Corticosterone $100 \mu \mathrm{M}$ \\
\hline Hedgehog pathway & - & 0.005 & 0.0002 \\
\hline GFR $\rightarrow$ NCOR2 signaling & + & 0.0001 & 0.00008 \\
\hline GFR $\rightarrow$ AP-I/CREB/CREBBP/ELK-SRF/MYC signaling & + & 0.0005 & 0.0006 \\
\hline Adherens junction regulation & + & 0.003 & 0.0003 \\
\hline Guanylate cyclase pathway & + & 0.004 & 0.0004 \\
\hline Apoptosis regulation & + & 0.003 & 0.003 \\
\hline Gonadotrope cell activation & + & 0.005 & 0.00003 \\
\hline
\end{tabular}

Neuropsychopharmacology 

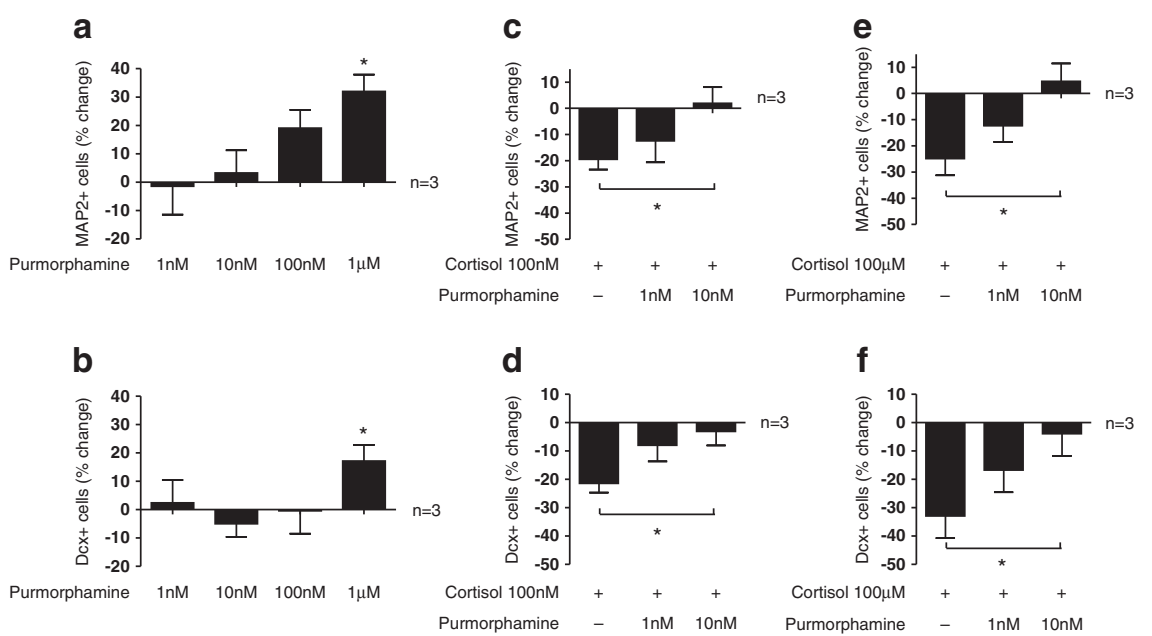

Figure 3 Effects of purmorphamine on neurogenesis. Purmorphamine increases neuronal differentiation into microtubule-associated protein 2 (MAP2)positive neurons (a) and into doublecortin (Dcx)-positive neuroblasts (b). The decrease in MAP2- and Dcx-positive cells upon treatment with the low cortisol concentration (I00 nM) is counteracted by purmorphamine $(10 \mathrm{~nm})(\mathrm{c}, \mathrm{d})$. The decrease in MAP2- and Dcx-positive cells upon treatment with the high cortisol concentration $(100 \mu \mathrm{M})$ is also counteracted by purmorphamine $(10 \mathrm{nM})$ (e, f). Three independent experiments were conducted on independent cultures $(n=3)$. Four wells were analyzed per treatment condition in each experiment and three random, non-overlapping pictures were analyzed for each well. All data are mean \pm s.e.m. $* P<0.05$, compared with the vehicle-treated control condition or as indicated.

activation of this pathway promotes neuronal differentiation (Ahn and Joyner, 2005; Cai et al, 2008), which, as described above, is decreased upon treatment with low and high cortisol concentrations in our experiments.

\section{Stimulation of Hedgehog Signaling Counteracts Cortisol Effects on Neurogenesis}

In order to test the hypothesis that inhibition of Hedgehog signaling is indeed part of the mechanism by which cortisol decreases neurogenesis, we co-treated cells with cortisol and with the smoothened (SMO)-agonist, purmorphamine, which activates Hedgehog signaling (Sinha and Chen, 2006). As expected, purmorphamine $(1 \mathrm{nM}-1 \mu \mathrm{M})$ increased neuronal differentiation into MAP2-positive neurons (Oneway ANOVA, $P=0.02, F_{1,4}=3.88, n=3$; Figure $3 \mathrm{a}$ ) and into Dcx-positive neuroblasts (One-way ANOVA, $P=0.13$, $F_{1,4}=2, \quad n=3 ; \quad t$-test: veh $v s$ Purmorphamine $1 \mu \mathrm{m}$, $P=0.03, n=3$; Figure $3 \mathrm{~b}$ ). Importantly, co-treatment with the two lowest concentrations of purmorphamine, which do not exert an effect on neuronal differentiation by themselves ( $1 \mathrm{~nm}$ and $10 \mathrm{~nm}$, see Figure $3 \mathrm{a}$ and $\mathrm{b}$ ), counteracted the decrease in MAP2- and Dcx-positive neurons upon treatment with $100 \mathrm{nM}$ (Figure 3c and d) and with $100 \mu \mathrm{m}$ of cortisol (Figure $3 \mathrm{e}$ and $\mathrm{f}$ ). These data support the notion that inhibition of Hedgehog signaling by cortisol significantly contributes to the decrease in neuronal differentiation.

\section{Regulation of Molecular Signaling Pathways in Prenatally Stressed Rats}

In order to compare our in vitro findings in human cells with an in vivo model of stress-induced biological and behavioral abnormalities, we conducted gene expression microarray and pathway analysis in the hippocampus of prenatally stressed (PNS) rats, a well established animal model of depression in which hippocampal neurogenesis is decreased (Lemaire et al, 2000; Pryce et al, 2011). Consistent
Table 2 Pathways Regulated by Prenatal Stress in the Rat Hippocampus

\begin{tabular}{lcc}
\hline Pathway name & Change & P-value \\
\hline NF-kB signaling & - & 0.018 \\
TGF $\beta$ Receptor $\rightarrow$ SMAD2/3-signaling & - & 0.026 \\
Insulin Action & + & 0.034 \\
Glycogen metabolism & + & 0.037 \\
Fatty acid oxidation & + & 0.046 \\
Hedgehog pathway & - & 0.047 \\
Leptin receptor $\rightarrow$ ELK-SRF signaling & + & 0.049 \\
ROS metabolism & - & 0.049 \\
\hline
\end{tabular}

with the literature, adult PNS rats (PND 62) exhibited significantly increased blood concentrations of CORT (control: $226 \pm 66 \mathrm{ng} / \mathrm{ml}, n=5$; PNS: $437 \pm 82 \mathrm{ng} / \mathrm{ml}, n=5$; $P=0.03$ ). Again, here we present the results of the pathway analysis (see Table 2), while the complete gene expression analysis is presented in Supplementary Figure 7, Supplementary Table 5 and in the Supplementary Results. Importantly, we found that, among the eight pathways regulated in the hippocampus of adult PNS rats $(P<0.05)$, TGF $\beta$-SMAD2/3- and Hedgehog signaling were both inhibited, which is the same effect that we observed in human hippocampal progenitor cells treated with high, stressrelevant concentrations of cortisol (described above).

\section{qPCR Validation of Affymetrix Findings}

To confirm the importance of Hedgehog signaling for cortisol and stress effects, both in vitro and in vivo, we analyzed expression of three key targets of Hedgehog signaling by qPCR: the transcription factor, Gli, the 
Hedgehog pathway activator, SMO, and the Hedgehoginteracting protein, HHIP1. Both, in cortisol-treated hippocampal progenitor cells and in the hippocampus of adult PNS rats, we found a significant reduction of Gli (CORT $100 \mathrm{nM}: 0.66 \pm 0.08$ fold, $P=0.02, n=3$, CORT $100 \mu \mathrm{M}$ : $0.71 \pm 0.07$ fold, $P=0.02, n=3$; PNS: $0.64 \pm 0.05$ fold, $P=0.006, n=5$ ) and SMO (CORT $100 \mathrm{nM}: 0.76 \pm 0.04$ fold, $P=0.03, n=3$, CORT $100 \mu \mathrm{M}: 0.55 \pm 0.1$ fold, $P=0.04$, $n=3$; PNS: $0.82 \pm 0.03, P=0.002, n=5$ ), and an increase in HHIP1, which inhibits Hedgehog signaling (CORT $100 \mathrm{nM}$ : $1.9 \pm 0.24$ fold, $P=0.03, n=3$, CORT $100 \mu \mathrm{M}: 2.4 \pm 0.36$ fold, $P=0.02, n=3$; PNS: $1.24 \pm 0.09$ fold, $P=0.04, n=5$ ). Together, these data confirm our finding that Hedgehog signaling is decreased upon cortisol treatment in human hippocampal progenitor cells and by PNS in the adult rat hippocampus.

In addition, we further validated 10 of the most significantly regulated genes with an absolute fold-change of $>1.3$ fold for each cortisol concentration $(100 \mathrm{nM}$ and $100 \mu \mathrm{M}$ ) and for PNS rats (Supplementary Tables 1 and 2). We found a high correlation of the fold-changes detected by affymetrix and by qPCR (cortisol-treated progenitor cells: Pearson's correlation: $r=0.92, \quad r^{2}=0.84, \quad P<0.0001$; Supplementary Figure 5b; Hippocampi of adult PNS rats: Pearson's correlation: $r=0.995, r^{2}=0.99, P<0.001$; Supplementary Figure 5c).

\section{DISCUSSION}

Here, we identify for the first time the molecular signaling pathways regulated by cortisol in human hippocampal progenitor cells and by PNS in the adult rat hippocampus. Firstly, we demonstrate that low and high cortisol concentrations exert differential MR- and GR-dependent effects on human hippocampal neurogenesis. We found that low cortisol concentrations, by predominantly activating the $\mathrm{MR}$, increase progenitor cell proliferation and shift neuronal development towards astrocyte development, while high concentrations of cortisol, by predominantly activating the GR, reduce both proliferation and neuronal differentiation, and inhibit the MR-induced increase in astrogliogenesis. At the molecular level, low cortisol concentrations enhance Notch/Hes-signaling, high cortisol concentrations inhibit TGF 3 -SMAD2/3-signaling, and both concentrations inhibit Hedgehog signaling. We also demonstrate that this inhibition of Hedgehog signaling is critically involved in the cortisol-induced decrease in neurogenesis. Secondly, and in line with the effects of high cortisol concentrations on human hippocampal progenitor cells, TGF 3 -SMAD2/3- and Hedgehog signaling are also downregulated in the hippocampus of adult prenatally stressed rats with elevated glucocorticoid levels. We are confident that these novel findings shed light on important molecular mechanisms underlying stress-related disorders, such as major depression.

The differential and common effects of MR- and GRactivation on proliferation and neuronal differentiation have never been shown in a human model, but have been suggested by previous studies in rodents. For example, GR activation by high glucocorticoid levels, as they occur upon stress and in depression, consistently decreases hippocampal cell proliferation and neuronal differentiation in vivo (Gould et al, 1991; David et al, 2009; Mayer et al, 2006) and in vitro (Kim et al, 2004; Yu et al., 2011). Importantly, $\mathrm{GR}^{+1}-$ mice with a $50 \%$ gene dose reduction in the whole body, and mice with a forebrain-specific deletion of the GR, show stress hypersensitivity and reduced hippocampal neurogenesis (Ridder et al, 2005; Kronenberg et al, 2009; Boyle et al, 2006), possibly caused by impaired hypothalamus-pituitary-adrenal (HPA) axis feedback inhibition and subsequent hypercortisolemia, affecting the hippocampus. Accordingly, mice overexpressing the GR are stress-resistant (Ridder et al, 2005). Interestingly, GR deletion in the entire nervous system causes increased CORT levels but does not elicit decreased hippocampal neurogenesis (Tronche et al, 1999; Gass et al, 2000). In contrast to the GR, MR activation by aldosterone increases hippocampal progenitor proliferation in adrenalectomized rats (Fischer et al, 2002), while MR knockout decreases proliferation (Gass et al, 2000), and the MR antagonist, spironolactone, increases neuronal differentiation (Chang et al, 2008). These animal studies, together with our human cellular data, therefore, support the notion that MR- and GR-activation indeed exert opposite effects on progenitor proliferation, while activation of both receptors decreases neuronal differentiation.

Interestingly, we identified several relevant molecular signaling mechanisms involved in the differential MR- and GR-mediated effects of cortisol on hippocampal neurogenesis and astrogliogenesis. The low cortisol concentration $(100 \mathrm{nM})$ activates Notch-/Hes-signaling, which has been shown by previous studies to increase progenitor proliferation and glia differentiation (Guo et al, 2009; Kageyama et al, 1999), an effect that we indeed observe in our experiments. In contrast, the high cortisol concentration downregulates Notch/Hes-signaling, thereby potentially inhibiting MR-mediated effects on proliferation and astrogliogenesis. Moreover, the high cortisol concentration inhibits TGF $\beta$-SMAD2/3-signaling, which has previously been shown to be decreased upon GR activation (Meisler et al, 1995; Battista et al, 2006) and in depressed patients (Musil et al, 2011). Furthermore, increased TGF $\beta$ signaling promotes neurogenesis in vitro and in vivo (Battista et al, 2006; Graciarena et al, 2010; Seuntjens et al, 2009), suggesting that the cortisol-induced inhibition of this pathway may potentially contribute to the GR-dependent decrease in neurogenesis in our cellular model.

Finally, both low and high concentrations of cortisol inhibit Hedgehog signaling. Hedgehog signaling promotes neuronal differentiation (Ahn et al, 2005; Cai et al, 2008; Kageyama et al, 1999) and inhibition of this pathway is therefore in line with decreased neurogenesis upon treatment with both low and high cortisol concentrations in our study. Indeed, we demonstrate that stimulating Hedgehog signaling with the pharmacological compound, purmorphamine, counteracts the cortisol-induced decrease in neurogenesis, supporting the notion that inhibition of this pathway may be significantly involved in the effects of cortisol on neurogenesis.

Importantly, in our experiments TGF $\beta$-SMAD2/3- and Hedgehog signaling are also downregulated in the hippocampus of adult rats with increased blood glucocorticoid levels as a result of PNS, an established animal model of 
depression (Fumagalli et al, 2007). This is the same effect that we observed in our cellular model upon treatment with high (stress-relevant) concentrations of cortisol, again indicating that common signaling pathways may be particularly relevant for stress- and glucocorticoid effects on the hippocampus, both in human cells and in rodent tissue. Indeed we found that three key targets of Hedgehog signaling-the transcription factor, Gli, the Hedgehog receptor, SMO, and the Hedgehog inhibitor, HHIP1-are regulated exactly in the same way by cortisol in vitro and by PNS in vivo. It is noteworthy, however, that despite these striking similarities, we have also found pathways and genes that were regulated differently by high cortisol concentrations in vitro and by stress in vivo, most likely due to the fact that the majority of cells in the hippocampus are neurons and glia cells, while progenitor cells only comprise a very small portion of the hippocampus. Accordingly, previous gene expression studies upon cortisol treatment in other cell types or tissues, such as fibroblasts, preadipocytes or fetal whole brain tissue, have found some similarities in gene expression when compared with our study, probably because cortisol was used as a common stimulus; but they also found many differences, likely due to tissue-specific effects of glucocorticoids (Smith et al., 2008; Bujalska et al, 2006; Salaria et al, 2006). In particular, cortisol-induced changes in Hedgehog signaling were not detected by these studies on other cell types, supporting the notion that the regulation of Hedgehog signaling may indeed be most relevant for the hippocampus and for hippocampal progenitor cells.

Are our findings relevant to the understanding of depression pathogenesis? Post-mortem studies have confirmed that patients with major depression-a condition often characterized by elevated glucocorticoids-show reduced hippocampal neurogenesis, while antidepressants reverse or prevent this effect (Boldrini et al, 2009, 2012; Lucassen et al, 2010). Moreover, we have shown that antidepressants reduce glucocorticoid actions in vitro using human hippocampal progenitor cells (Anacker et al, 2011b), as well as in vivo using electroencephalography (Pariante et al., 2011) and neuroendocrine tests (Pariante, 2003). Therefore, the prevailing model suggests that excessive MRand GR-stimulation by hypercortisolemia reduces neurogenesis in the hippocampus of depressed patients, which can be rescued by effective antidepressant treatment. It is, however, important to emphasize that the 'neurogenesis hypothesis' of depression is not universally accepted. For example, some studies have questioned a potential functional role for hippocampal neurogenesis, because relatively few new neurons are being added to the adult brain (Rakic 1985). Furthermore, one human post-mortem brain study did not find any changes in hippocampal cell proliferation in depressed patients, although the study sample was heterogeneous and comprised depressed and antidepressant-treated patients (Reif et al, 2006). Moreover, rodent studies have suggested that reduced neurogenesis per se may indeed not contribute to the development of depression, while being critical for the effects of antidepressants (Santarelli et al, 2003; Surget et al, 2008). However, more recent studies have demonstrated that neurogenesis significantly contributes to the regulation of the HPA axis and depressive behavior in rodents (Snyder et al, 2011; Surget et al, 2011), suggesting that reduced neurogenesis may impair hippocampal inhibitory control over the HPA axis during stress, subsequently causing sustained hypercortisolemia and depressive symptoms (Anacker and Pariante 2012).

A possible limitation of our in vitro model is that the immortalized human fetal hippocampal progenitor cells, while being invaluable for our understanding of the molecular mechanisms in the human hippocampus, may differ from the situation in a adult organism in vivo. Moreover, the cortisol concentrations used in our in vitro experiments are likely higher than the so far unknown physiological concentrations in the brain of stressed or depressed humans. It is important to emphasize that the differences between the in vivo cortisol and in vitro CORT concentrations in our study may be explained by the high abundance of albumin in the cell culture media and the reportedly high expression of $11 \beta$-hydroxysteroid dehydrogenase 2 and multidrug-resistant p-glycoprotein in fetal tissue and stem cells (Brown et al, 1996; Islam et al, 2005, Lin et al, 2006). These factors likely diminish cortisol bioavailability in our progenitor cell system, thereby making potentially higher concentrations necessary to specifically activate corticosteroid receptors. Indeed, our dose-response study of GR activation shows that the GR is not saturated at the high cortisol concentration $(100 \mu \mathrm{M})$. A further clear limitation of the in vitro model employed in this study is that no data is yet available on electrophysiological properties of differentiated HPC03A/07 cells. It will thus be an important line of future investigation to examine whether HPC03A/07 cells develop electrophysiological properties of functionally mature neurons, in order to fully characterize the potential impact of this cellular model for functional neuronal network properties and human neurogenesis in vitro.

In conclusion, our data shed light on the effects of cortisol on human hippocampal neurogenesis and astrogliogenesis, and identify novel molecular signaling pathways involved in the effects of glucocorticoids and stress on the hippocampus. Targeting these pathways may provide future treatment strategies to counteract cortisol-induced impairments in hippocampal plasticity in depressed patients.

\section{ACKNOWLEDGEMENTS}

Dr Anacker was funded by a studentship from the NIHR 'Biomedical Research Centre for Mental Health', Institute of Psychiatry and South London and Maudsley NHS Foundation Trust, London, UK; Dr Zunszain was supported by a NARSAD Young Investigator Award. Dr Thuret was supported in part by a grant from Research Councils UK. Dr Cirulli was supported by the Italian Ministry of Health (Ricerca finalizzata 2009). Dr Riva was funded by Regione Lombardia (Accordo Quadro 2010). Dr Gennarelli was supported by the Italian Ministry of Health (Ricerca Corrente) and Regione Lombardia (ID:17387Sal-13). Dr Pariante was funded by a Clinician Scientist Fellowship from the Medical Research Council, UK (G108/603) and a grant from the Commission of European Communities 7th Framework Programme Collaborative Project Grant Agreement n 22963 (Mood Inflame). The authors would like to 
thank Dr A. Vernon, King's College London, for kindly providing the purmorphamine aliquots.

\section{DISCLOSURE}

$\mathrm{J}$ Price acted as a consultant and received payment from ReNeuron Group within the last 2 years. CM Pariante has received fees as a speaker or as a member of advisory board, as well as research funding, from pharmaceutical companies that commercialize or are developing antidepressants in the last 3 years, such as Lilly, Servier, and Janssen. MA Riva has received compensation as speaker/consultant for AstraZeneca, Bristol-Myers Squibb, Eli Lilly, Servier, Takeda. PA Zunszain has received speaker fees from Servier. All other authors have no potential conflict of interest to declare.

\section{REFERENCES}

Ahn S, Joyner AL (2005). In vivo analysis of quiescent adult neural stem cells responding to Sonic hedgehog. Nature 437: 894-897.

Anacker C, Pariante CM (2012). Can adult neurogenesis buffer stress responses and depressive behaviour? Mol Psychiatry 17: 9-10.

Anacker C, Zunszain PA, Carvalho LA, Pariante CM (2011a). The glucocorticoid receptor: pivot of depression and of antidepressant treatment? Psychoneuroendocrinology 36: 415-425.

Anacker C, Zunszain PA, Cattaneo A, Carvalho LA, Garabedian MJ, Thuret $S$ et al (2011b). Antidepressants increase human hippocampal neurogenesis by activating the glucocorticoid receptor. Mol Psychiatry 16: 738-750.

Battista D, Ferrari CC, Gage FH, Pitossi FJ (2006). Neurogenic niche modulation by activated microglia: transforming growth factor beta increases neurogenesis in the adult dentate gyrus. Eur J Neurosci 23: 83-93.

Boldrini M, Underwood MD, Hen R, Rosoklija GB, Dwork AJ, John Mann J et al (2009). Antidepressants increase neural progenitor cells in the human hippocampus. Neuropsychopharmacology 34: 2376-2389.

Boyle MP, Kolber BJ, Vogt SK, Wozniak DF, Muglia LJ (2006). Forebrain glucocorticoid receptors modulate anxiety-associated locomotor activation and adrenal responsiveness. J Neurosci 26: 1971-1978.

Brown RW, Diaz R, Robson AC, Kotelevtsev YV, Mullins JJ, Kaufman MH et al (1996). The ontogeny of 11 beta-hydroxysteroid dehydrogenase type 2 and mineralocorticoid receptor gene expression reveal intricate control of glucocorticoid action in development. Endocrinology 137: 794-797.

Bujalska IJ, Quinkler M, Tomlinson JW, Montague CT, Smith DM, Stewart PM (2006). Expression profiling of 11beta-hydroxysteroid dehydrogenase type-1 and glucocorticoid-target genes in subcutaneous and omental human preadipocytes. J Mol Endocrinol 37: 327-340.

Cai C, Thorne J, Grabel L (2008). Hedgehog serves as a mitogen and survival factor during embryonic stem cell neurogenesis. Stem Cells 26: 1097-1108.

Chang YT, Chen YC, Wu CW, Yu L, Chen HI, Jen CJ et al (2008). Glucocorticoid signaling and exercise-induced downregulation of the mineralocorticoid receptor in the induction of adult mouse dentate neurogenesis by treadmill running. Psychoneuroendocrinology 33: 1173-1182.

Datson NA, Speksnijder N, Mayer JL, Steenbergen PJ, Korobko O, Goeman J et al (2010). The transcriptional response to chronic stress and glucocorticoid receptor blockade in the hippocampal dentate gyrus. Hippocampus 22: 359-371.
David DJ, Samuels BA, Rainer Q, Wang JW, Marsteller D, Mendez I et al (2009). Neurogenesis-dependent and -independent effects of fluoxetine in an animal model of anxiety/depression. Neuron 62: 479-493.

De Kloet ER, Derijk R (2004). Signaling pathways in brain involved in predisposition and pathogenesis of stress-related disease: genetic and kinetic factors affecting the MR/GR balance. Ann $N$ Y Acad Sci 1032: 14-34.

Fischer AK, von Rosenstiel P, Fuchs E, Goula D, Almeida OF, Czeh B (2002). The prototypic mineralocorticoid receptor agonist aldosterone influences neurogenesis in the dentate gyrus of the adrenalectomized rat. Brain Res 947: 290-293.

Fujioka A, Fujioka T, Ishida Y, Maekawa T, Nakamura S (2006). Differential effects of prenatal stress on the morphological maturation of hippocampal neurons. Neuroscience 141: 907-915.

Fumagalli F, Molteni R, Racagni G, Riva MA (2007). Stress during development: Impact on neuroplasticity and relevance to psychopathology. Prog Neurobiol 81: 197-217.

Gass P, Kretz O, Wolfer DP, Berger S, Tronche F, Reichardt HM et al (2000). Genetic disruption of mineralocorticoid receptor leads to impaired neurogenesis and granule cell degeneration in the hippocampus of adult mice. EMBO Rep 1: 447-451.

Gould E, Woolley CS, Cameron HA, Daniels DC, McEwen BS (1991). Adrenal steroids regulate postnatal development of the rat dentate gyrus: II. Effects of glucocorticoids and mineralocorticoids on cell birth. J Comp Neurol 313: 486-493.

Graciarena M, Depino AM, Pitossi FJ (2010). Prenatal inflammation impairs adult neurogenesis and memory related behavior through persistent hippocampal TGFbetal downregulation. Brain Behav Immun 24: 1301-1309.

Guo YJ, Zhang ZJ, Wang SH, Sui YX, Sun Y (2009). Notch1 signaling, hippocampal neurogenesis and behavioral responses to chronic unpredicted mild stress in adult ischemic rats. Prog Neuropsychopharmacol Biol Psychiatry 33: 688-694.

Islam MO, Kanemura Y, Tajria J, Mori H, Kobayashi S, Shofuda T et al (2005). Characterization of $\mathrm{ABC}$ transporter $\mathrm{ABCB} 1$ expressed in human neural stem/progenitor cells. FEBS Lett 579: 3473-3480.

Kageyama R, Ohtsuka T (1999). The Notch-Hes pathway in mammalian neural development. Cell Res 9: 179-188.

Karalay O, Doberauer K, Vadodaria KC, Knobloch M, Berti L, Miquelajauregui A et al (2011). Prospero-related homeobox 1 gene (Prox1) is regulated by canonical Wnt signaling and has a stage-specific role in adult hippocampal neurogenesis. Proc Natl Acad Sci USA 108: 5807-5812.

Kim JB, Ju JY, Kim JH, Kim TY, Yang BH, Lee YS et al (2004). Dexamethasone inhibits proliferation of adult hippocampal neurogenesis in vivo and in vitro. Brain Res 1027: 1-10.

Kronenberg G, Kirste I, Inta D, Chourbaji S, Heuser I, Endres M et al (2009). Reduced hippocampal neurogenesis in the GR(+/-) genetic mouse model of depression. Eur Arch Psychiatry Clin Neurosci 259: 499-504.

Lemaire V, Koehl M, Le Moal M, Abrous DN (2000). Prenatal stress produces learning deficits associated with an inhibition of neurogenesis in the hippocampus. Proc Natl Acad Sci USA 97: 11032-11037.

Lin T, Islam O, Heese K (2006). ABC transporters, neural stem cells and neurogenesis - a different perspective. Cell Research 16: 857-871.

Lucassen PJ, Stumpel MW, Wang Q, Aronica E (2010). Decreased numbers of progenitor cells but no response to antidepressant drugs in the hippocampus of elderly depressed patients. Neuropharmacology 58: 940-949.

Matrisciano F, Busceti CL, Bucci D, Orlando R, Caruso A, Molinaro $\mathrm{G}$ et al (2011). Induction of the Wnt antagonist Dickkopf-1 is involved in stress-induced hippocampal damage. PLoS one 6: e16447. 
Mayer JL, Klumpers L, Maslam S, de Kloet ER, Joels M, Lucassen PJ (2006). Brief treatment with the glucocorticoid receptor antagonist mifepristone normalises the corticosterone-induced reduction of adult hippocampal neurogenesis. J Neuroendocrinol 18: 629-631.

Meisler N, Shull S, Xie R, Long GL, Absher M, Connolly JP et al (1995). Glucocorticoids coordinately regulate type I collagen pro alpha 1 promoter activity through both the glucocorticoid and transforming growth factor beta response elements: a novel mechanism of glucocorticoid regulation of eukaryotic genes. J Cell Biochem 59: 376-388.

Montaron MF, Piazza PV, Aurousseau C, Urani A, Le Moal M, Abrous DN (2003). Implication of corticosteroid receptors in the regulation of hippocampal structural plasticity. Eur J Neurosci 18: 3105-3111.

Mootha VK, Lindgren CM, Eriksson KF, Subramanian A, Sihag S, Lehar J et al (2003). PGC-1alpha-responsive genes involved in oxidative phosphorylation are coordinately downregulated in human diabetes. Nat Genet 34: 267-273.

Musil R, Schwarz MJ, Riedel M, Dehning S, Cerovecki A, Spellmann I et al (2011). Elevated macrophage migration inhibitory factor and decreased transforming growth factor-beta levels in major depression-no influence of celecoxib treatment. J Affect Disord 134: 217-225.

Pariante CM, Alhaj HA, Arulnathan VE, Gallagher P, Hanson A, Massey E et al (2011). Central glucocorticoid receptor-mediated effects of the antidepressant, citalopram, in humans: A study using EEG and cognitive testing. Psychoneuroendocrinology 37: 618-628.

Pariante CM, Lightman SL (2008). The HPA axis in major depression: classical theories and new developments. Trends Neurosci 31: 464-468.

Pariante CM, Miller AH (2001). Glucocorticoid receptors in major depression: relevance to pathophysiology and treatment. Biol Psychiatry 49: 391-404.

Pryce CR, Aubert Y, Maier C, Pearce PC, Fuchs E (2011). The developmental impact of prenatal stress, prenatal dexamethasone and postnatal social stress on physiology, behaviour and neuroanatomy of primate offspring: studies in rhesus macaque and common marmoset. Psychopharmacology (Berl) 214: 33-53.

Rakic P (1985). Limits of neurogenesis in primates. Science 227: 1054-1056.

Reif A, Fritzen S, Finger M, Strobel A, Lauer M, Schmitt A et al (2006). Neural stem cell proliferation is decreased in schizophrenia, but not in depression. Mol Psychiatry 11: 514-522.

Ridder S, Chourbaji S, Hellweg R, Urani A, Zacher C, Schmid W et al (2005). Mice with genetically altered glucocorticoid receptor expression show altered sensitivity for stress-induced depressive reactions. J Neurosci 25: 6243-6250.

Salaria S, Chana G, Caldara F, Feltrin E, Altieri M, Faggioni F et al (2006). Microarray analysis of cultured human brain aggregates following cortisol exposure: implications for cellular functions relevant to mood disorders. Neurobiol Dis 23: 630-636.

Santarelli L, Saxe M, Gross C, Surget A, Battaglia F, Dulawa S et al (2003). Requirement of hippocampal neurogenesis for the behavioral effects of antidepressants. Science 301: 805-809.
Seuntjens E, Umans L, Zwijsen A, Sampaolesi M, Verfaillie CM, Huylebroeck D (2009). Transforming Growth Factor type beta and Smad family signaling in stem cell function. Cytokine Growth Factor Rev 20: 449-458.

Shull S, Meisler N, Absher M, Phan S, Cutroneo K (1995). Glucocorticoid-induced down regulation of transforming growth factor-beta 1 in adult rat lung fibroblasts. Lung 173: 71-78.

Sinha S, Chen JK (2006). Purmorphamine activates the Hedgehog pathway by targeting Smoothened. Nat Chem Biol 2: 29-30.

Sippel M, Rajala R, Korhonen L, Bornhauser B, Sokka AL, Naito M et al (2009). Dexamethasone regulates expression of BRUCE/ Apollon and the proliferation of neural progenitor cells. FEBS Lett 583: 2213-2217.

Smith JC, Boone BE, Opalenik SR, Williams SM, Russell SB (2008). Gene profiling of keloid fibroblasts shows altered expression in multiple fibrosis-associated pathways. J Invest Dermatol 128: $1298-1310$

Snyder JS, Soumier A, Brewer M, Pickel J, Cameron HA (2011). Adult hippocampal neurogenesis buffers stress responses and depressive behaviour. Nature 476: 458-461.

Surget A, Saxe M, Leman S, Ibarguen-Vargas Y, Chalon S, Griebel $\mathrm{G}$ et al (2008). Drug-dependent requirement of hippocampal neurogenesis in a model of depression and of antidepressant reversal. Biol Psychiatry 64: 293-301.

Surget A, Tanti A, Leonardo ED, Laugeray A, Rainer Q, Touma C et al (2011). Antidepressants recruit new neurons to improve stress response regulation. Mol Psychiatry 16: 1177-1188.

Tronche F, Kellendonk C, Kretz O, Gass P, Anlag K, Orban PC et al (1999). Disruption of the glucocorticoid receptor gene in the nervous system results in reduced anxiety. Nat Genet 23: 99-103.

Tukey JW (1977). Exploratory Data Analysis. Reading MA, Addison-Wesley: Cambridge.

Wong EY, Herbert J (2006). Raised circulating corticosterone inhibits neuronal differentiation of progenitor cells in the adult hippocampus. Neuroscience 137: 83-92.

Xi G, Zhang X, Zhang L, Sui Y, Hui J, Liu S et al (2010). Fluoxetine attenuates the inhibitory effect of glucocorticoid hormones on neurogenesis in vitro via a two-pore domain potassium channel, TREK-1. Psychopharmacology (Berl) 214: 747-759.

Yu S, Yang S, Holsboer F, Sousa N, Almeida OF (2011). Glucocorticoid regulation of astrocytic fate and function. PLoS one 6: e22419.

Zunszain PA, Anacker C, Cattaneo A, Choudhury S, Musaelyan K, Myint AM et al (2011). Interleukin-1beta: a new regulator of the kynurenine pathway affecting human hippocampal neurogenesis. Neuropsychopharmacology 37: 939-949.

Zunszain PA, Anacker C, Cattaneo A, Carvalho LA, Pariante CM (2011). Glucocorticoids, cytokines and brain abnormalities in depression. Prog Neuropsychopharmacol Biol Psychiatry 35: 722-729.

(i) (-) $\odot$ This work is licensed under a Creative Commons Attribution-NonCommercial-NoDerivs 3.0 Unported License. To view a copy of this license, visit http:// creativecommons.org/licenses/by-nc-nd/3.0/

Supplementary Information accompanies the paper on the Neuropsychopharmacology website (http://www.nature.com/npp) 